\title{
Erratum: Magnetic field dependence of the thermopower of Kondo-correlated quantum dots: Comparison with experiment [Phys. Rev. B 100, 155126 (2019)]
}

\author{
T. A. Costi $®$
}

(Received 26 November 2021; published 9 December 2021)

DOI: 10.1103/PhysRevB.104.239901

There is a typographical error in the third line of the caption to Fig. 1(b): $\varepsilon_{0}=-3 \Gamma$ should read $\varepsilon_{0}=-1.5 \Gamma$ (as in the legends). There is also a factor of 2 error in the calculated thermocurrent $G S$ ( $G S / 2$ was calculated) which affects the theoretical GS shown in Fig. 6 (left four panels), Fig. 9, and Fig. 13 (right four panels). The error is corrected in the figures below. The conclusions are unaffected.

We thank M. A. Manya, G. B. Martins, and M. S. Figuiera for drawing our attention to the above.

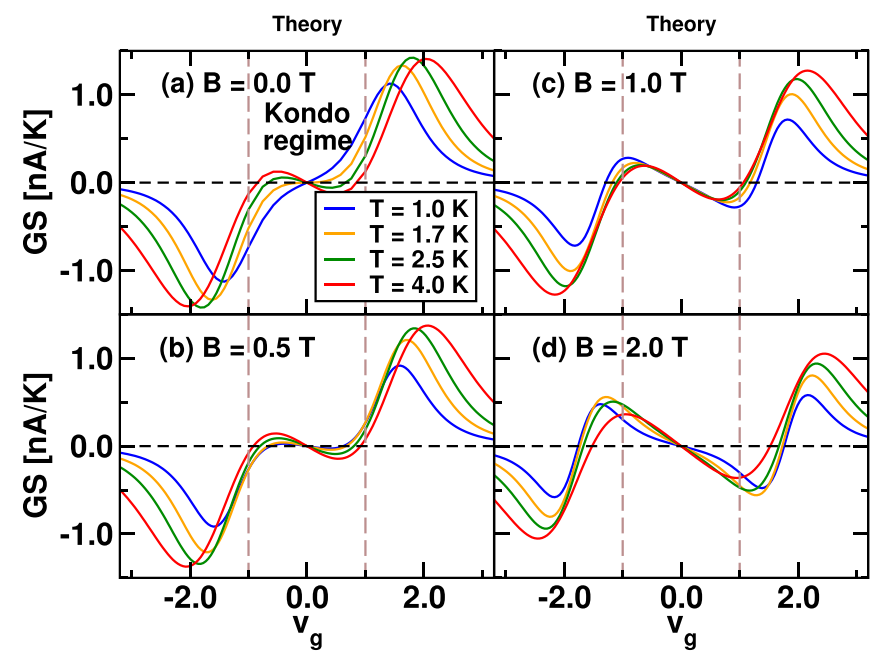

FIG. 6. (Left four panels) revised plot for $G S$ correcting a factor of two ( $G S / 2$ was plotted in the paper).

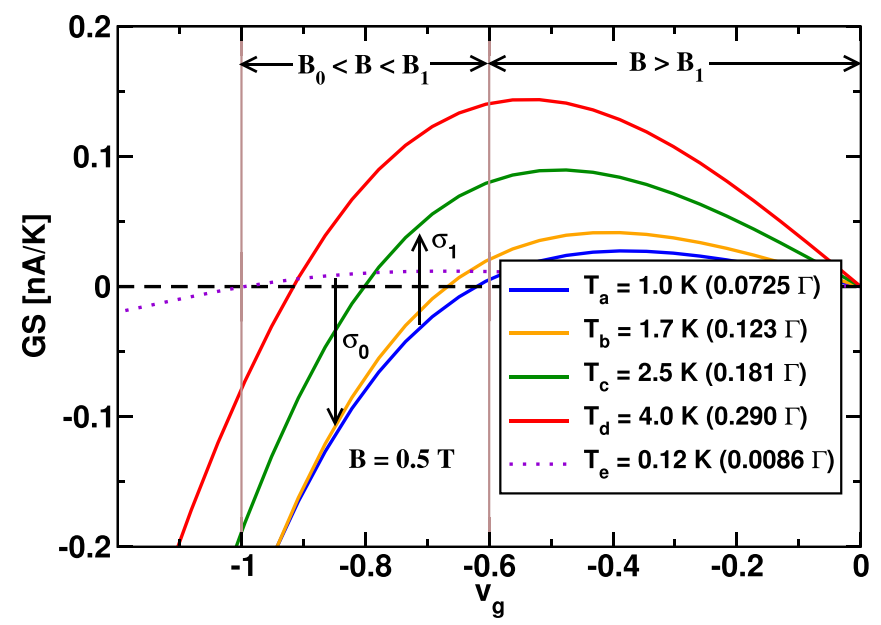

FIG. 9. Revised plot for $G S$ correcting a factor of two $(G S / 2$ was plotted in the paper).

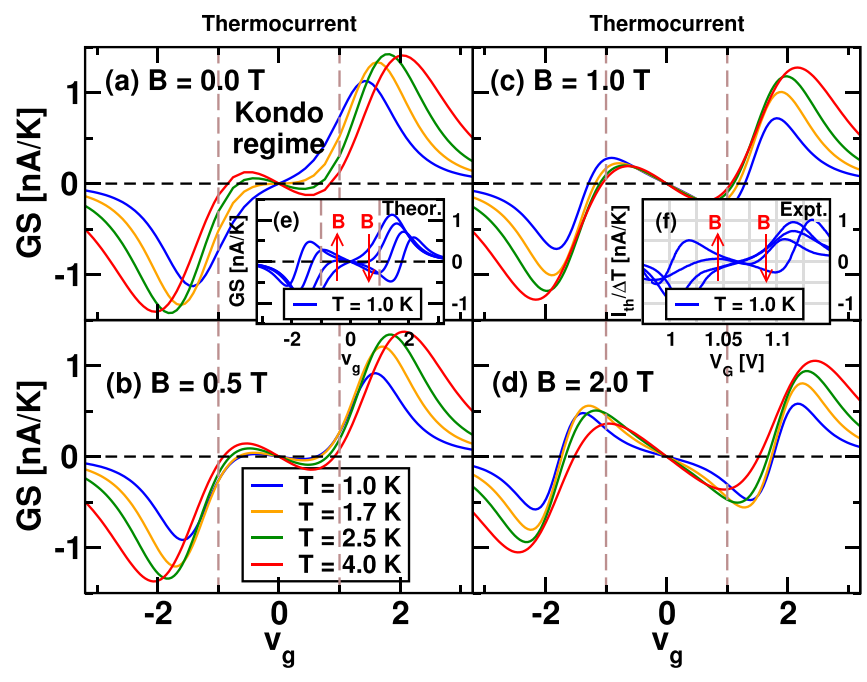

FIG. 13. (Right four panels) revised plot for $G S$ correcting a factor of two $(G S / 2$ was plotted in the paper). 